\title{
Search for short-period variable stars in the open cluster NGC 2301
}

\author{
S.-L. Kim ${ }^{1}$, M.-Y. Chun ${ }^{1}$, B.-G. Park ${ }^{1}$, S. H. Lee ${ }^{2}$, H. Sung ${ }^{1}$, \\ H. B. Ann ${ }^{2}$, M. G. Lee ${ }^{3}$, Y.-B. Jeon ${ }^{1}$, and I.-S. Yuk ${ }^{1}$ \\ 1 Korea Astronomy Observatory, Taejon, 305-348, Korea \\ 2 Department of Earth Science, Pusan National University, Pusan, 609-735, Korea \\ 3 Astronomy Program, SEES, Seoul National University, Seoul, 151-742, Korea
}

Received 28 June 2000 / Accepted 13 March 2001

\begin{abstract}
Time-series CCD photometric observations of an intermediate age open cluster NGC 2301 were performed to search for variable stars, particularly $\gamma$ Dor stars and $\delta$ Sct stars. We also carried out $U B V I$ photometry to study physical properties of variable stars in the cluster. We derived observational parameters of the cluster from $U B V I$ photometric results. The best fit of the empirical ZAMS in a color-color diagram gives a reddening value of $E(B-V)=0.05 \pm 0.03$. We also estimated a distance modulus of $\left(V-M_{V}\right)_{0}=9.6 \pm 0.1$ and an age of $\log t=8.4 \pm 0.1$, from the ZAMS and isochrone fitting in a color-magnitude diagram. Using $V$ time-series data, we carefully examined light variations of 923 stars fainter than $10 .{ }^{\mathrm{m}} 0$, and discovered nine short-period variable stars; two $\gamma$ Dor candidates, five eclipsing binaries, one Slowly Pulsating $B$ star and one magnetic Chemically Peculiar variable candidate. Two $\gamma$ Dor candidates with relatively short periods of around 0.3 days are located in a redder region (about 0.1 in $B-V$ color) than the red edge of $\delta$ Sct instability strip in the color-magnitude diagram.
\end{abstract}

Key words. open cluster: NGC 2301 - stars: C-M diagrams - stars: variables: $\gamma$ Dor - stars: binaries: eclipsing

\section{Introduction}

Open clusters are obviously important targets to investigate physical characteristics of Pop I pulsating variable stars such as $\delta$ Sct stars, $\gamma$ Dor stars and Slowly Pulsating B stars (hereafter, called SPBs). Simultaneous photometry of variable and comparison stars in the cluster with a CCD enables us to obtain more precise time-series data by observing the stars under the same weather and equal instrumental conditions. Furthermore, since all the cluster members are assumed to have the same interstellar reddening, distance, age and chemical abundance, we can put strong constraints on these physical parameters of the cluster variables. The asteroseismological importance of CCD observations for $\delta$ Sct stars in open clusters was described in an extensive review by Kjeldsen (2000).

$\gamma$ Dor stars are a new class of pulsating variable stars. Careful investigations of the proto-type star $\gamma$ Dor were carried out only recently by the SAAO group (Balona et al. 1994a, 1994b and 1996), although it was discovered to be a variable long ago by Cousins \& Warren (1963). They are main-sequence or sub-giant (luminosity class V or IV) stars with spectral types ranging from A7 to F5, near the red edge of the $\delta$ Sct instability strip in the

Send offprint requests to: S.-L. Kim, e-mail: slkim@kao.re.kr
HR diagram (Handler 1999; Kaye et al. 1999). They have pulsation periods of $0.3 \sim 3.0$ and amplitudes less than $0^{\mathrm{m}} 1$ in $V$ passband. On the basis of photometric and spectroscopic observation results, their light variations are recognized as excitation of high-order low-degree nonradial g-mode oscillations. From an asteroseismological point of view, the g-mode oscillations seen in $\gamma$ Dor stars are very important. Since they penetrate deeper into the stellar interior than p-mode oscillations do, they provide us with more valuable information on the inner stellar structure. $\gamma$ Dor stars have similar observational properties (pulsation amplitude and position in a color-magnitude diagram, etc.) as $\delta$ Sct stars, except for the large difference of pulsation periods. $\delta$ Sct stars have very short periods of $0.02 \sim$ 0.2 and spectral types ranging from A3 to F0. Their variability is known to be caused by the excitation of p-mode oscillations.

So far, few $\gamma$ Dor stars in open clusters have been reported; eight candidates in NGC 2516 (Zerbi et al. 1998), two candidates in M 34 (Krisciunas \& Patten 1999) and another two candidates in the Pleiades cluster (Martín \& Rodríguez 2000). From the fact that no $\gamma$ Dor stars have been found in the relatively old Hyades cluster and several candidates have been detected in the young open clusters mentioned above, Krisciunas \& Patten (1999) suggested 


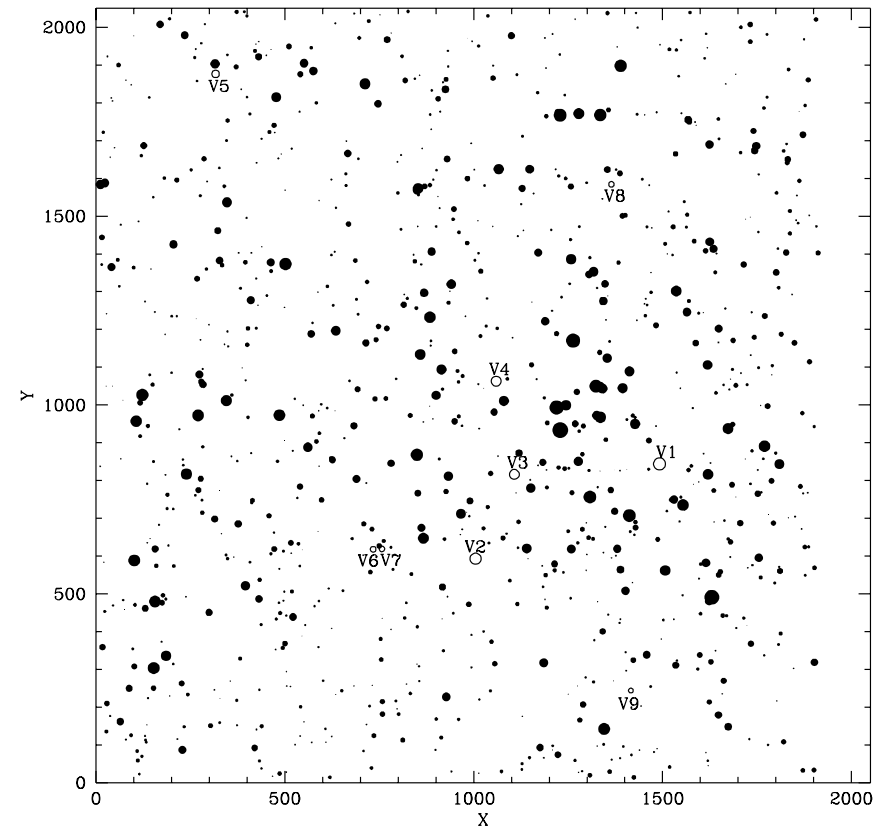

Fig. 1. Observed CCD field $\left(11^{\prime} \cdot 6 \times 11^{\prime} \cdot 6\right)$ of the open cluster NGC 2301. North is up and east is to the left. Nine new variable stars are marked by open circles and their ID numbers

that $\gamma$ Dor type phenomena occur in stars younger than about 250 Myr. However, Handler (1999) noted that several $\gamma$ Dor stars exist far from the ZAMS, i.e., they have rather old ages. He proposed instead that, on the basis of metallicity distribution for bona-fide $\gamma$ Dor stars and prime candidates, metallicity could play a role in $\gamma$ Dor type variability. No $\gamma$ Dor stars have been reported in relatively metal-rich clusters such as the Hyades cluster.

In this paper, we present the time-series CCD photometric results of the open cluster NGC $2301\left(\mathrm{RA}_{2000}=\right.$ $\left.6^{\mathrm{h}} 51^{\mathrm{m}} 8, \quad \mathrm{DEC}_{2000}=0^{\circ} 28^{\prime} ; l=212.56, \quad b=0.29\right)$. NGC 2301 is an ideal target for detecting new $\gamma$ Dor and $\delta$ Sct stars, because there are a number of stars near the $\delta$ Sct instability strip in the HR diagram and most of the probable member stars in the cluster are moderately concentrated within our CCD field (about 12 arcmin). The main purpose of this study is to establish the occurrence of $\gamma$ Dor type variability in the open cluster with an intermediate age about $250 \mathrm{Myr}$ and solar metal abundance.

\section{Observation and data reduction}

Time-series observations were made at the $1.8 \mathrm{~m}$ telescope of Bohyunsan Optical Astronomy Observatory. We obtained a total of $200 \mathrm{~V}$ frames for five nights (19.3 hours monitoring) from February 6th to 13th, 1999, with a SITe $2048 \times 2048$ CCD. Typical photometric seeing $(F W H M)$ was less than 2 .' 0 . Exposure times were chosen in a range of $20 \mathrm{~s}$ to $40 \mathrm{~s}$, depending on seeing and atmospheric transparency. In order to minimize position-dependent external errors (Frandsen et al. 1989), we carefully controlled the telescope by locating a star at the same position in CCD frames during our observing run. We also carried out
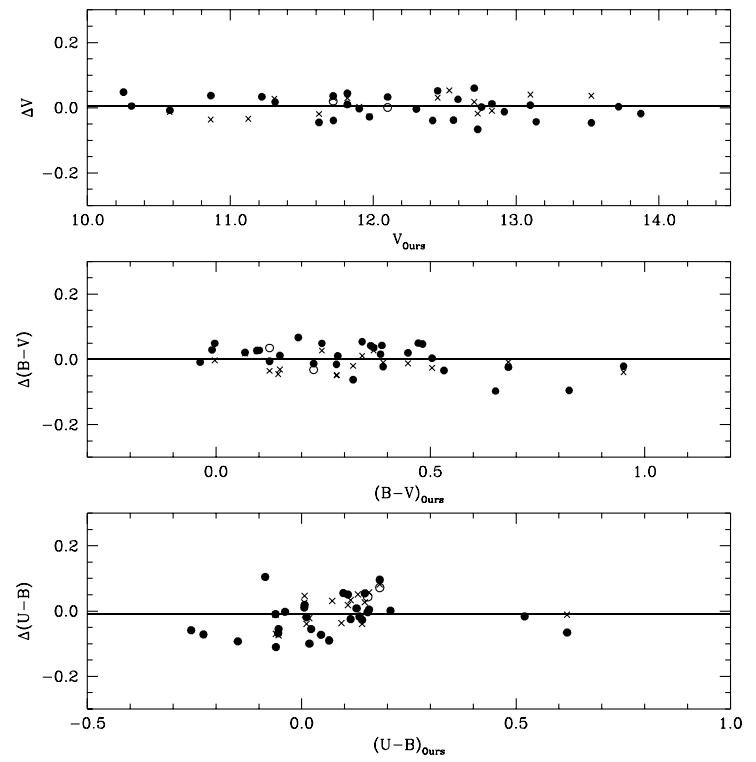

Fig. 2. Magnitude and color differences between our data and the previous ones by Hoag et al. (1961; crosses), Grubissich \& Purgathofer (1962; open circles) and Chun (1997; filled circles)

$U B V I$ photometry on a photometric night (March 13), in order to estimate the physical parameters of the cluster. The Johnson $U B V$ and Cousins $I$ filters were used. Several tens of $U B V I$ standard stars in Landolt (1992) were observed to transform instrumental magnitudes and colors onto the standard system.

Using the IRAF/CCDRED package, we processed the CCD images to correct overscan regions, trim unreliable subsections, subtract bias frames and correct the pixel-topixel inhomogeneity of quantum efficiency (flat fielding). Instrumental magnitudes were obtained via the empirical PSF (point spread function) fitting method using the photometric package in IRAF/DAOPHOT (Massey \& Davis 1992).

From photometric data of standard stars, we derived the following transformation equations;

$U=u+19.808+0.262(U-B)-0.619 X, \sigma_{U}=0{ }^{\mathrm{m}} 049$

$B=b+22.729+0.127(B-V)-0.390 X, \sigma_{B}=0.034$

$V=v+23.081-0.053(B-V)-0.268 X, \sigma_{V}=0 .{ }^{\mathrm{m}} 029$

$I=i+23.169+0.037(V-I)-0.154 X, \sigma_{I}=0 .{ }^{\mathrm{m}} 022$

where $U, B, V$ and $I$ are standard magnitudes, and $u$, $b, v$ and $i$ are instrumental magnitudes. The airmass is denoted by $X$.

After correcting for the difference between large aperture for standard stars (sparse fields) and small aperture for stars in the cluster (dense fields), we obtained the standard magnitudes and colors of the stars in NGC 2301 using the above equations. The observed 944 stars in the cluster are shown in Fig. 1. Our UBVI CCD photometric results are in good agreement with the previous photoelectric data (Hoag et al. 1961; Grubissich \& Purgathofer 1962) and CCD data (Chun 1997); differences between ours and the previous results are $\Delta V=0.004 \pm 0.031$, 


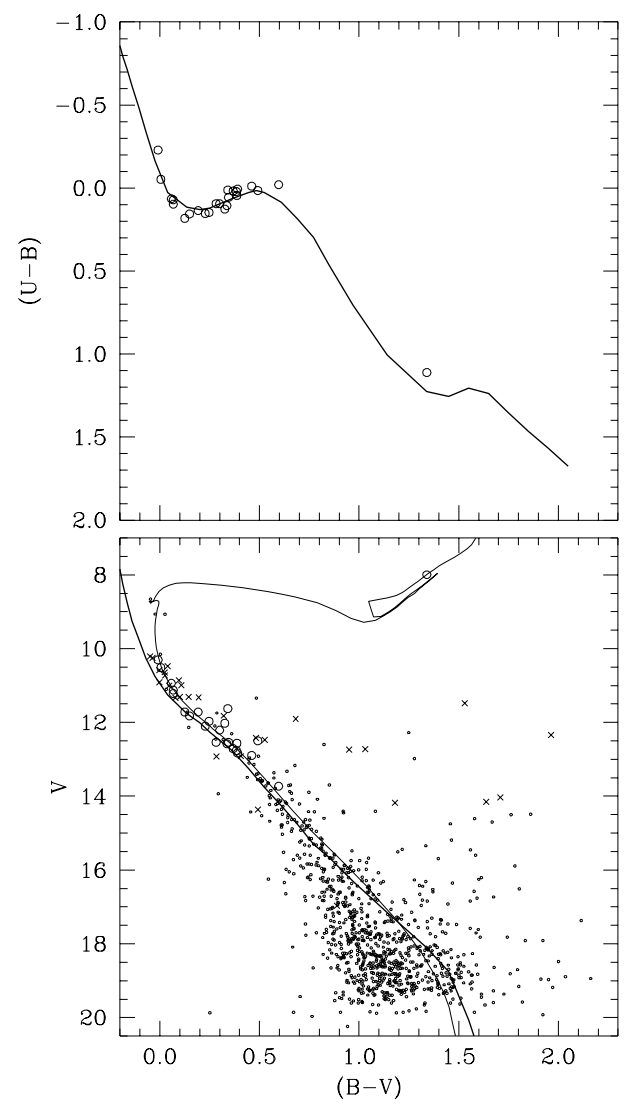

NGC 2301

$\mathrm{E}(\mathrm{B}-\mathrm{V})=0.05 \pm 0.03$

$\left(\mathrm{V}-\mathrm{M}_{\mathrm{V}}\right)_{0}=9.6 \pm 0.1$

$\mathrm{E}(\mathrm{U}-\mathrm{B}) / \mathrm{E}(\mathrm{B}-\mathrm{V})=0.72$

$\mathrm{E}(\mathrm{V}-\mathrm{I}) / \mathrm{E}(\mathrm{B}-\mathrm{V})=1.25$

$\mathrm{A}_{\mathrm{V}} / \mathrm{E}(\mathrm{B}-\mathrm{V})=3.1$

ZAMS : Sung \& Bessell(1999)

$\log$ Age $($ year $)=8.4(\mathrm{Z}=0.019)$

Isochrone : Girardi et al.(2000)

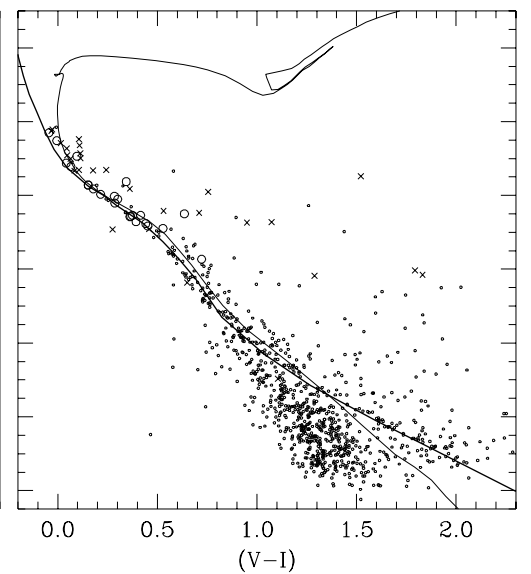

Fig. 3. Color-color and color-magnitude diagrams of NGC 2301. Thick solid lines denote the ZAMS and thin lines represent theoretical isochrone for $\log t=8.4$ and $Z=0.019$ model. Large open circles: probable members $\left(P_{\mu} \geq 0.7\right)$ and crosses: non-members, identified by Marie (1992) and Aiad (1986)

$\Delta(B-V)=-0.001 \pm 0.038, \Delta(U-B)=-0.008 \pm 0.054$ (Fig. 2). Our photometric data will be available in electronic form in the open cluster database (WEBDA, Mermilliod 1992).

We applied an ensemble normalization technique (Gilliland \& Brown 1988) in order to normalize instrumental magnitudes of the time-series $V$ frames. Firstly, we chose a few tens of normalization stars which are unsaturated bright stars ranging from $12^{\mathrm{m}} \cdot 0$ to $14^{\mathrm{m}} 0$, not variable and not in the edge of a CCD frame. Then, coefficients of the following equation were calculated for each time-series CCD frame;

$V=v+a_{1}+a_{2}(B-V)+a_{3} X+a_{4} Y$,

where $V$ and $v$ are standard magnitude and instrumental magnitude for normalization stars, respectively. Here $X$ and $Y$ are coordinate values of stars in a CCD frame. Finally, we normalized all observed stars for each CCD frame using the above equation. With this technique, we corrected color $\left(a_{2}\right)$ and position $\left(a_{3}\right.$ and $\left.a_{4}\right)$ dependent terms of the observation system and/or atmospheric differential extinction for all time-series CCD frames.

\section{Physical parameters}

Color-color and color-magnitude diagrams of the open cluster NGC 2301 are shown in Fig. 3. A number of

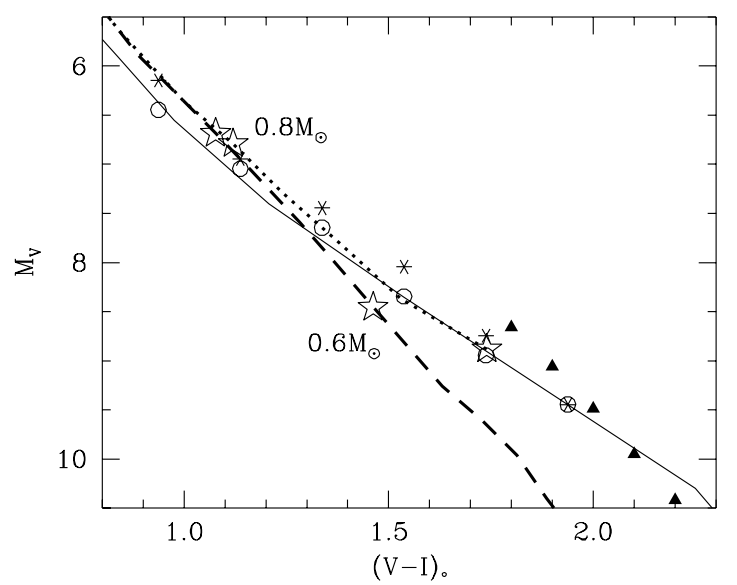

Fig. 4. Comparison of the empirical ZAMS (thin solid line) with new Padova theoretical isochrones (Girardi et al. 2000; thick dashed line) and the previous one (Bertelli et al. 1994; thick dotted line), for the solar metal abundance. We plotted the main-sequence fiducial point of NGC 2301 (this study; open circles), M 67 (Montgomery et al. 1993; asterisks) and NGC 2420 (von Hippel \& Gilmore 2000; filled triangles). The star symbols represent the initial mass of 0.6 and $0.8 M_{\odot}$ for the above two theoretical isochrones

field stars appeared below the main-sequence but relatively well-separated from the empirical ZAMS given by 


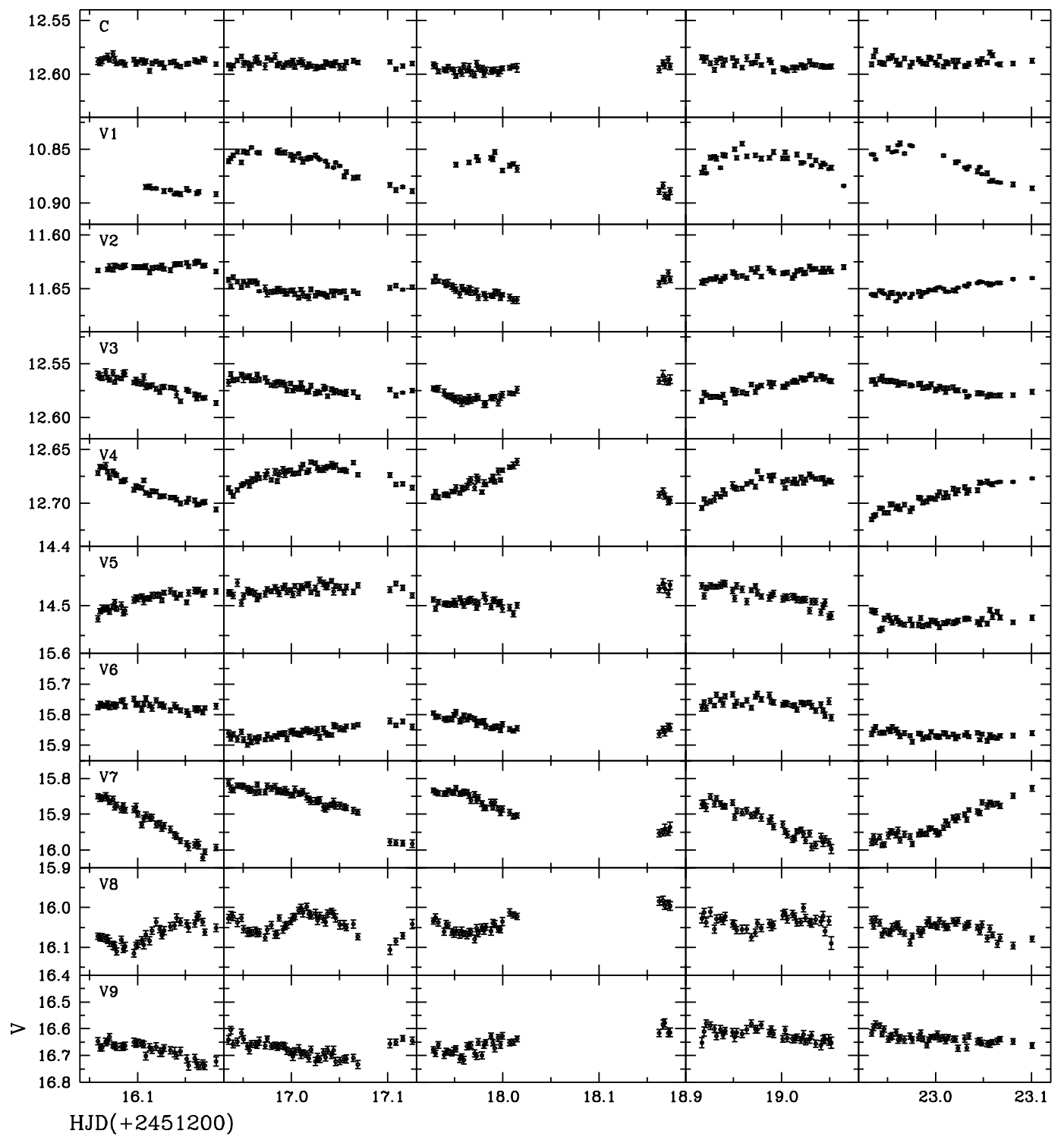

Fig. 5. Light variations of nine new variable stars in the observed field of NGC 2301. The error bar represents photometric error for each observation data. The brightness of a non-variable star (C) is also plotted for comparison

Sung \& Bessell (1999). In order to avoid the severe field star contamination (Mohan \& Sagar 1988) and obtain more accurate physical parameters of the cluster, we collected the membership data from the open cluster database (WEBDA, Mermilliod 1992). We assumed that those stars that have a membership probability $\left(P_{\mu}\right)$ less than 0.7 , from the proper motion study by Marie (1992), are cluster non-members.

The best fit of the empirical ZAMS in the color-color diagram gives a reddening value of $E(B-V)=0.05 \pm 0.03$, with a value of $E(U-B) / E(B-V)=0.72$. A reddening value of $E(V-I)=0.06 \pm 0.03$ was used, adopting $E(V-I) / E(B-V)=1.25$ (Dean et al. 1978). We obtained a distance modulus of $\left(V-M_{V}\right)_{0}=9.6 \pm 0.1$ and an age of $\log t=8.4 \pm 0.1$, from the ZAMS and isochrone fit in the color-magnitude diagram, assuming $R_{V}=3.1$. Considering the observed value of $[\mathrm{Fe} / \mathrm{H}]=0.06$ (Twarog et al. 1997; DDO photometry) and $[\mathrm{Fe} / \mathrm{H}]=0.04$
Table 1. Physical parameters of the open cluster NGC 2301

\begin{tabular}{lccc}
\hline Reference & $E(B-V)\left(V-M_{V}\right)_{0} \log$ Age $(\mathrm{yr})^{\dagger}$ \\
\hline Nissen (1988) & $0 . \cdot 036$ & 9.62 & \\
Mohan \& Sagar (1988) & 0.04 & 9.5 & \\
Napiwotzki et al. (1991) & 0.05 & 9.2 & 8.3 \\
Twarog et al. (1997) & $0 .{ }^{\mathrm{m}} 04$ & $9 . \cdot 68$ & \\
This study & 0.05 & 9.6 & $8.4 \pm 0.1$ \\
\hline
\end{tabular}

${ }^{\dagger}$ : Estimated from stellar models with convective overshooting.

(Nissen 1988; uvby- $\beta$ photometry), we made use of the theoretical isochrone with the solar metal abundance $(Z=$ 0.019) by Girardi et al. (2000). The isochrone had been derived from stellar models with convective overshooting. 

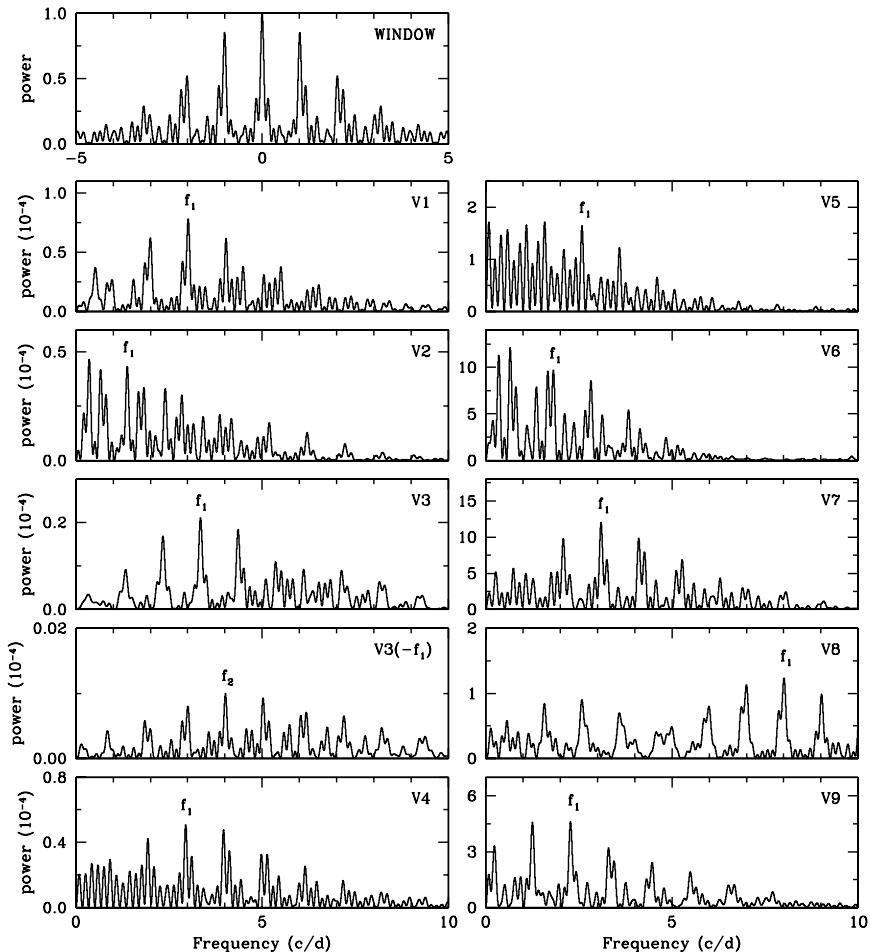

Fig. 6. Power spectra of nine new variable stars. The spectral window is shown in the top panel

Physical parameters obtained in this study are consistent with the previous results (Table 1).

It might be noted that new isochrone model of the Padova group (Girardi et al. 2000) is more poorly fit with the empirical ZAMS and the observed data for NGC 2301 (this study), M 67 (Montgomery et al. 1993) and NGC 2420 (von Hippel \& Gilmore 2000) than the previous model given by Bertelli et al. (1994), in the low-mass region less than about $0.8 M_{\odot}$ (Fig. 4). For a given initial mass, the new isochrones put the sequence to the brighter and bluer regions in a color-magnitude diagram than the previous ones.

\section{Variable stars}

With $V$-band time-series CCD data, we examined light variations of 923 stars among 944 stars in the observed field. The saturated stars brighter than $10^{\mathrm{m}}$ and the stars located within 20 pixels from edge of the time-series frames were excluded because they could result in unreliable photometric results. Especially, for bright stars $\left(10^{\mathrm{m}} \sim 13^{\mathrm{m}}\right)$ near $\delta$ Sct instability strip, we investigated the signal/noise amplitude ratio $(S / N)$ of a frequency with the biggest power in a power spectrum of each star, in order to check for small amplitude variations. The $S / N$ ratio has been used as the detection criterion of pulsation frequencies; e.g. Paparo et al. (2000)'s analysis for a $\delta$ Sct star 57 Tau. We set the pulsation frequency criterion with the $S / N$ ratio greater than 4.0 (Breger et al. 1993).

We discovered nine short-period variable stars in the observed field of NGC 2301. A finding chart and light
Table 2. Results of frequency analysis for new variable stars

\begin{tabular}{ccrrrr}
\hline ID & $\begin{array}{c}\text { Frequency } \\
(\mathrm{c} / \mathrm{d})\end{array}$ & $\begin{array}{r}\text { Amplitude } \\
(\mathrm{mmag})\end{array}$ & Phase $^{1}$ & $S / N^{2}$ & $\begin{array}{r}\text { s.d. }^{3} \\
(\mathrm{mmag})\end{array}$ \\
\hline V1 & $f_{1}=3.015$ & 18.4 & 1.80 & 12.7 & 4.5 \\
V2 & $f_{1}=1.378$ & 11.0 & 2.38 & 5.2 & 5.6 \\
V3 & $f_{1}=3.344$ & 9.4 & -1.01 & 19.0 & 2.7 \\
& $f_{2}=4.014$ & 2.2 & -0.70 & 4.1 & \\
V4 & $f_{1}=2.946$ & 16.4 & 2.10 & 7.9 & 4.9 \\
V5 & $f_{1}=2.584$ & 21.7 & 3.64 & 5.5 & 13.2 \\
V6 & $f_{1}=1.817$ & 58.6 & 0.83 & 12.0 & 16.1 \\
V7 & $f_{1}=3.095$ & 68.8 & -0.21 & 8.4 & 19.3 \\
V8 & $f_{1}=8.008$ & 23.8 & 0.85 & 4.1 & 18.8 \\
V9 & $f_{1}=2.276$ & 35.9 & 1.54 & 4.5 & 22.3 \\
\hline
\end{tabular}

${ }^{1} V=$ Const. $+\Sigma_{j} A_{j} \cos \left\{2 \pi f_{j}\left(t-t_{0}\right)+\phi_{j}\right\}, t_{0}=$ HJD 2451200.

${ }^{2}$ Signal to noise ratio, introduced by Breger et al. (1993).

${ }^{3}$ Standard deviation, fitting synthetic curves to the data.

variations of variable stars are shown in Figs. 1 and 5 , respectively. We derived the periods of variable stars from the discrete Fourier analysis (Scargle 1989). Figure 6 shows power spectra and Table 2 lists detailed results of the analysis. Five variable stars (V1, V4, V7, V8 and V9) have one dominant frequency with the largest power, $3.015,2.946,3.095,8.008$ and $2.276 \mathrm{c} / \mathrm{d}$, respectively. We detected two frequencies for V3 from the multiple frequency analysis (Kim \& Lee 1996). For the other three stars, V2, V5 and V6, several peaks are shown in the low frequency region so that the period determination seems to be complicated. It could be an effect of non-sinusoidal light variations and our short-time baseline data in comparison with their long periods. Furthermore, our data suffered from a severe aliasing problem as shown in the window spectrum. Although we chose a frequency to show the most reasonable light curve after examining phase diagrams for frequencies with large power, the periods of three stars, particularly for V2, might be uncertain.

Phase diagrams of nine variable stars are shown in Fig. 7. The orbital periods of the five eclipsing binary stars (V5 V9) are two times the periods calculated from their frequencies. During the phase-match process (Hoffmeister et al. 1985), we made small adjustments of the periods for several stars to show good curves. The light curve of $\mathrm{V} 2$ shows great difference with those of pulsating stars or eclipsing binaries, and might be similar to those of magnetic CP (Chemically Peculiar) variable stars HD 36668 (Adelman 2000) and HD 35298 (Adelman \& Rice 1999). Observational characteristics viz. spectral type (A5; Mermilliod 1992), low amplitude ( $\left.\sim 0^{\mathrm{m}} 03\right)$ and period $(0 \mathrm{~d} 741)$ support the possibility that V2 is a magnetic CP variable candidate (Schöneich \& Želwanowa 1993 for a review of variability among magnetic CP stars). Spectroscopic observation is required to prove its chemical peculiarity. However, since the period of V2 is ambiguous (see above), the characteristics of V2 remain unclear. From the shape of their light curves, we could classify five stars 

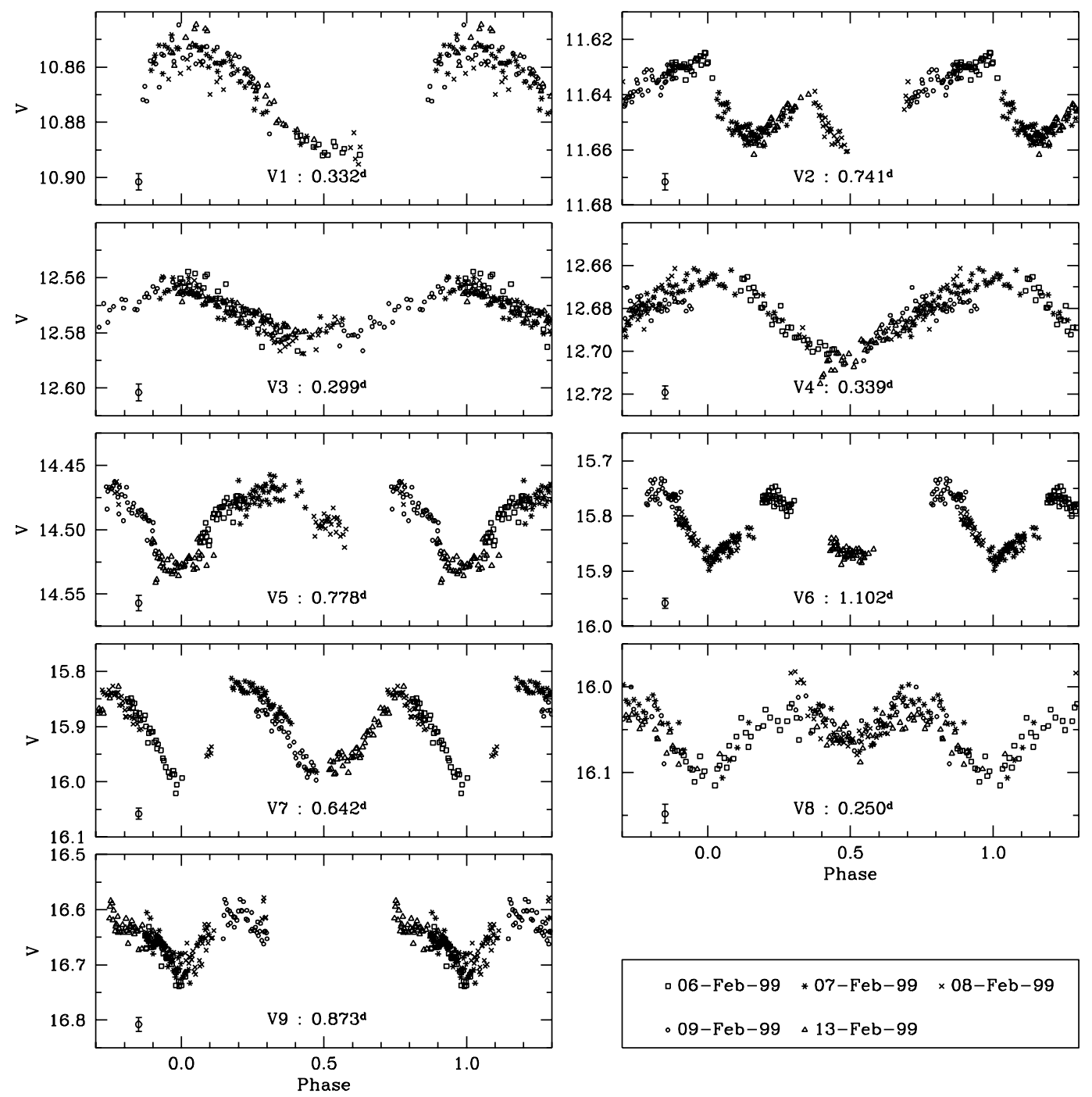

Fig. 7. Phase diagrams of nine variable stars discovered in this study. Data points obtained over five nights are differently marked for each observation night. Typical observational errors are given by error bars in the lower left part of each diagram

of V5 V V as W UMa or $\beta$ Lyr type eclipsing binaries (Hoffmeister et al. 1985). V5 might be a field eclipsing binary because it is brighter by about 1. 5 than the ZAMS.

Considering their positions in a color-magnitude diagram (Fig. 8), shapes of light curve and pulsation frequencies, we classified V1 as SPB (see Waelkens et al. 1998; Choi et al. 1999), and V3 and V4 as $\gamma$ Dor candidates. V1 might be a field SPB star because its cluster membership is zero (Mermilliod 1992) and its location is redder by about $0{ }^{\mathrm{m}} 08$ than the red boundary of theoretical SPB instability strip (Choi et al. 1999). Pulsation periods of two $\gamma$ Dor candidates seem to be relatively short in comparison with those of the other $\gamma$ Dor stars; the shortest period among $\gamma$ Dor candidates has been known to be 0.339 for H1284 in the Pleiades cluster (Martín \& Rodríguez 2000). However, these periods are too long to classify them as $\delta$ Sct stars (Fig. 9) and the positions of two $\gamma$ Dor candidates in the color-magnitude diagram are redder (about $0^{\mathrm{m}} 1$ in $B-V$ color) than the red edge of the $\delta$ Sct instability strip (Fig. 8). Spectroscopic evidence is needed to confirm the status of the new $\gamma$ Dor candidates. Our results are summarized in Table 3.

It should be noted that, among 16 stars within the $\delta$ Sct instability strip, we could not detect any $\delta$ Sct type variability at a detection level of about $3 \mathrm{mmag}$. The low incidence of $\delta$ Sct stars in NGC 2301 is similar to those of four open clusters investigated by Viskum et al. (1997). The incidence is much lower than $\sim 30 \%$ of field stars (Breger 1979) and $\sim 20 \%$ of the open cluster NGC 6134 at a detection level of 1 mmag (Frandsen et al. 1996).

\section{Conclusion}

We present the discovery of one field SPB star, two $\gamma$ Dor candidates, five eclipsing binaries and one magnetic CP variable candidate in the open cluster NGC 2301. Our results confirm that $\gamma$ Dor type phenomena occur in stars with an intermediate age of $250 \mathrm{Myr}(\log t=8.4)$ and solar metal abundance. However, it might be difficult to identify whether it is age (Krisciunas \& Patten 1999) or 
Table 3. Basic parameters of nine variable stars discovered in NGC 2301

\begin{tabular}{|c|c|c|c|c|c|c|c|c|c|}
\hline ID OUR & $\mathrm{ID}_{\mathrm{BDA}}^{\dagger}$ & $V$ & $B-V$ & Period & Max. $\Delta V$ & Epoch $^{\ddagger}$ & Type & $P_{\mu}^{\dagger}$ & Remark \\
\hline $\mathrm{V} 1$ & 38 & 10.864 & 0.095 & 0.332 & $\sim 0.04$ & 2451216.97 & SPB star & 0.00 & non-member \\
\hline $\mathrm{V} 2$ & 45 & $11^{\mathrm{m}} 621$ & $0 .{ }^{\mathrm{m}} 341$ & 0.741 & $\sim 0.03$ & 2451216.17 & Magnetic CP star (?) & 0.91 & \\
\hline V3 & 50 & 12.562 & $0 .{ }^{\mathrm{m}} 387$ & 0.299 & $\sim 0.02$ & 2451216.06 & $\gamma$ Dor star & 0.96 & multi-frequencies \\
\hline $\mathrm{V} 4$ & 56 & $12^{\mathrm{m}} \cdot 708$ & $0 .{ }^{\mathrm{m}} 368$ & 0.339 & $\sim 0.04$ & 2451216.02 & $\gamma$ Dor star & 0.93 & \\
\hline V5 & 1733 & $14^{\mathrm{m}} 452$ & $0 . \mathrm{m}^{\mathrm{m}} 922$ & 0.778 & $\sim 0.08$ & 2451216.01 & Eclipsing binary & - & non-member (?) \\
\hline V6 & - & $15 \cdot 872$ & $0 .{ }^{\mathrm{m}} 925$ & 1. 102 & $\sim 0.15$ & 2451216.95 & Eclipsing binary & - & \\
\hline V7 & - & $15^{\mathrm{m}} \cdot 975$ & $0 .{ }^{\mathrm{m}} 910$ & 0.642 & $\sim 0.17$ & 2451216.18 & Eclipsing binary & - & \\
\hline V8 & 1668 & 16.075 & $1 .{ }^{\mathrm{m}} 097$ & 0.250 & $\sim 0.09$ & 2451216.09 & Eclipsing binary & - & \\
\hline V9 & 1323 & 16.605 & $1 .{ }^{\mathrm{m}} 041$ & 0.873 & $\sim 0.12$ & 2451216.17 & Eclipsing binary & - & \\
\hline
\end{tabular}

${ }^{\dagger}$ : Identification and membership probability from the open cluster database by Mermilliod (1992).

‡: Maximum brightness for pulsating stars and minimum for eclipsing binary stars.

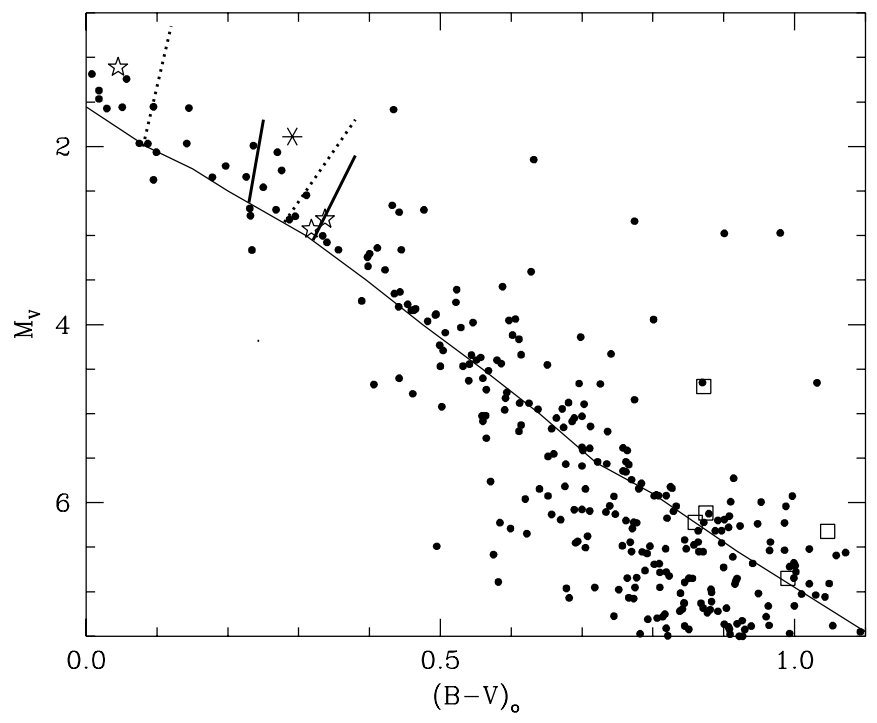

Fig. 8. Position of new variable stars in a color-magnitude diagram of NGC 2301. Three pulsating stars $(2 \gamma$ Dor stars and $1 \mathrm{SPB})$ are denoted by star symbols and five eclipsing binaries, open squares. A magnetic $\mathrm{CP}$ variable candidate is represented as an asterisk. Thin solid line represents the ZAMS (Sung \& Bessell 1999). Thick dotted line and thick solid line are the borders of $\delta$ Sct instability strip (Breger 1979) and $\gamma$ Dor instability strip (Handler 1999), respectively. The reddening and distance modulus derived in this study were used

metallicity (Handler 1999) that controls $\gamma$ Dor type variability. No $\delta$ Sct type variable was detected among 16 stars within the $\delta$ Sct instability strip of NGC 2301. More systematic studies to search for $\delta$ Sct and $\gamma$ Dor stars in open clusters with a wide range of age and metallicity would be necessary in order to understand what kinds of physical parameters control pulsation phenomena of p-mode oscillation ( $\delta$ Sct stars) and g-mode oscillation $(\gamma$ Dor stars). Further spectroscopic observations should be needed to confirm variable characteristics of two $\gamma$ Dor candidates and one magnetic CP candidate.

Acknowledgements. We thank Dr. P. Lampens for her valuable advice and Dr. Y.-J. Moon for his careful reading. One

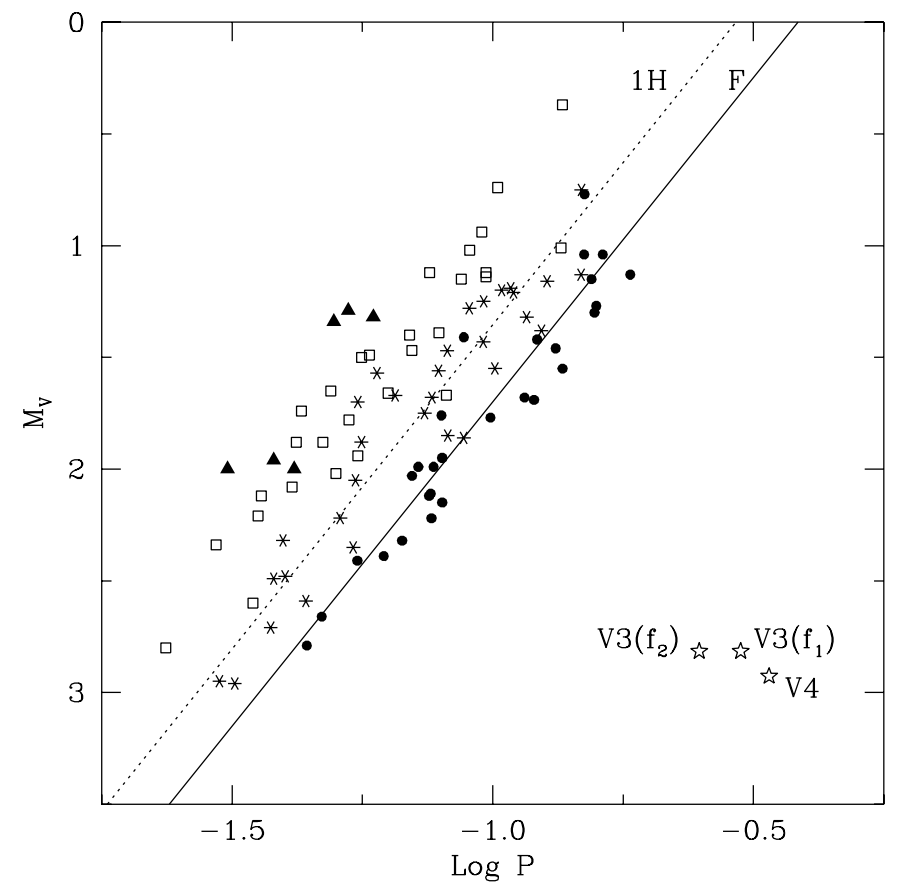

Fig. 9. Period-luminosity relation of $\delta$ Sct stars. Filled circles: F-mode, asterisks: $1 \mathrm{H}$-mode, open squares: $2 \mathrm{H}$-mode, and filled triangles: 3H-mode, identified by Rodríguez et al. (1994). Solid and dashed lines represent the empirical period-luminosity relation of $\delta$ Sct stars for F-mode and $1 \mathrm{H}$-mode oscillation, respectively (Fernie 1992). The periods of two $\gamma$ Dor candidates discovered in this study (star symbols) are longer than the F-mode oscillation period of $\delta$ Sct stars

of the authors (M. G. Lee) was partially supported by the Brain Korea 21 Project. This work was partly supported by the MOST (Ministry of Science and Technology) Research Fund.

\section{References}

Adelman, S. J. 2000, A\&A, 357, 548

Adelman, S. J., \& Rice, R. H. 1999, A\&AS, 136, 111

Aiad, A. 1986, Bull. Fac. Sci. Cairo Univ., 54, 729

Balona, L. A., Hearnshaw, J. B., Koen, C., et al. 1994a, MNRAS, 267, 103 
Balona, L. A., Krisciunas, K., \& Cousins, A. W. J. 1994b, MNRAS, 270, 905

Balona, L. A., Böhm, T., Foing, B. H., et al. 1996, MNRAS, 281,1315

Bertelli, G., Bressan, A., Chiosi, C., Fagotto, F., \& Nasi, E. 1994, A\&AS, 106, 275

Breger, M. 1979, PASP, 91, 5

Breger, M., Stich, J., Garrido, R., et al. 1993, A\&A, 271, 482

Choi, H. S., Kim, S.-L., Kang, Y. H., \& Park, B.-G. 1999, A\&A, 348,789

Chun, M. Y. 1997, Ph.D. Thesis, Seoul National Univ.

Cousins, A. W. J., \& Warren, P. R. 1963, Mon. Notes Astron. Soc. S. Afr., 22, 65

Dean, J. F., Warren, P. R., \& Cousins, A. W. J. 1978, MNRAS, 183,569

Fernie, J. D. 1992, AJ, 103, 1647

Frandsen, S., Dreyer, P., \& Kjeldsen, H. 1989, A\&A, 215, 287

Frandsen, S., Balona, L. A., Viskum, M., Koen, C., \& Kjeldsen, H. 1996, A\&A, 308, 132

Gilliland, R. L., \& Brown, T. M. 1988, PASP, 100, 754

Girardi, L., Bressan, A., Bertelli, G., \& Chiosi, C. 2000, A\&AS, 141,371

Grubissich, C., \& Purgathofer, A. 1962, Z. Ap., 54, 41

Handler, G. 1999, MNRAS, 309, L19

Hoag, A. A., Johnson, H. L., Iriarte, B., et al. 1961, Publications of the United States Naval Observatory, vol. XVII, Part VII

Hoffmeister, C., Richter, G., \& Wenzel, W. 1985, in Variable stars, 15, 201

Kaye, A. B., Handler, G., Krisciunas, K., Porreti, E., \& Zerbi, F. M. 1999, PASP, 111, 840

Kjeldsen, H. 2000, in The impact of large-scale surveys on pulsating star research, ed. L. Szabados, \& D. W. Kurtz, PASPC, 203, 415
Kim, S.-L., \& Lee, S.-W. 1996, A\&A, 310, 831

Krisciunas, K., \& Patten, B. M. 1999, Inf. Bull. Variable Stars, No. 4705

Landolt, A. U. 1992, AJ, 104, 340

Marie, M. A. 1992, A\&SS, 198, 121

Martín, S., \& Rodríguez, E. 2000, A\&A, 358, 287

Massey, P., \& Davis, L. E. 1992, A User's Guide to Stellar CCD Photometry with IRAF

Mermilliod, J. C. 1992, in Open cluster data base, BDA (http://obswww.unige.ch/webda)

Mohan, V., \& Sagar, R. 1988, Bull. Astr. Soc. India, 16, 159

Montgomery, K. A., Marschall, L. A., \& Janes, K. A. 1993, AJ, 106,181

Napiwotzki, R., Schönberner, D., \& Weidemann, V. 1991, A\&A, 243, L5

Nissen, P. E. 1988, A\&A, 199, 146

Paparo, M., Rodríguez, E., McNamara, B. J., et al. 2000, A\&AS, 142,1

Rodríguez, E., López de Coca, P., Rolland, A., Garrido, R., \& Costa, V. 1994, A\&AS, 106, 21

Scargle, J. D. 1989, ApJ, 343, 874

Schöneich, W., \& Želwanowa, E. 1993, in Peculiar versus normal phenomena in A-type and Related stars, ed. M. M. Dworetsky, et al., PASPC, 44, 327

Sung, H., \& Bessell, M. S. 1999, MNRAS, 306, 361

Twarog, B. A., Ashman, K. M., \& Anthony-Twarog, B. J. 1997, AJ, 114, 2556

von Hippel, T., \& Gilmore, G. 2000, AJ, 120, 1384

Viskum, M., Hernández, M. M., Belmonte, J. A., \& Frandsen, S. 1997, A\&A, 328, 158

Waelkens, C., Aerts, C., Kestens, E., Grenon, M., \& Eyer, L. 1998, A\&A, 330, 215

Zerbi, F. M., Mantegazza, L., Campana, S., \& Antonello, E. 1998, PASP, 110, 804 